\title{
Passive control of the flutter instability on a two-degrees-of-freedom system with pseudoelastic shape-memory alloy springs.
}

\author{
A. Malher ${ }^{1, a}$, O. Doaré ${ }^{1}$, C. Touzé ${ }^{1}$ \\ Unité de mécanique (UME), ENSTA-ParisTech, 828 Boulevard des Maréchaux, 91762 Palaiseau Cedex, France
}

\begin{abstract}
A passive control of aeroelastic instabilities on a two-degrees-of-freedom (dofs) system is considered here using shape memory alloys (SMA) springs in their pseudo-elastic regime. SMA present a solid-solid phase change that allow them to face strong deformations $(\sim 10 \%)$; in the pseudo-elastic regime, an hysteresis loop appears in the stress-strain relationship which in turn gives rise to an important amount of dissipated energy. This property makes the SMA a natural candidate for damping undesired vibrations in a passive manner. A 2 dofs system is here used to model the classical flutter instability of a wing section in an uniform flow. The SMA spring is selected on the pitch mode in order to dissipate energy of the predominant motion. A simple model for the SMA hysteresis loop is introduced, allowing for a quantitative study of the important parameters to optimize in view of an experimental design.
\end{abstract}

\section{Introduction}

Aeroelastic instabilities are an important issue in aeronautics, especially regarding the wing motions. Indeed, for a coupled system airflow - flexible structure, like aircraft wing, turbojet or bridge, a limit velocity exists above which the flexible structure cannot evacuate the energy received from the airflow anymore, then giving rise to strong or even fatal deformations. These instabilities, usually described under the generic term flutter instability, result from interaction between aerodynamic, inertial and elastic forces [1]. In this contribution, we focus on a passive control device for mitigating the flutter instability by using springs composed of shape memory alloys (SMA). In their pseudoelastic regime, SMA are known for showing the ability of dissipating an important amount of energy thanks to the hysteresis loop appearing in their stress-strain relationship, and has thus already been used in numerous applications ranging from civil engineering, aeronautics to medical industry [2,3]. Recent contributions have considered the dynamical responses of SMA springs from the theoretical viewpoint [4-6] in order to properly quantify the most proeminent features of the vibrations of simple single dof systems. Experimentally, a torsion pendulum has been recently used in order to clearly exhibit the softening effect of SMA oscillators [7]. The aim of this paper is to investigate the effect of a SMA spring on the flutter instability. More particularly, the most relevant parameters of a pseudo-elastic regime on the amplitudes of the limit cycle oscillations (LCO) will be analyzed, in order to quantify the effect of the dissipation brought by the hysteresis loop. The airfoil is modeled using the classical 2dofs system coupling pitch and heave motions [8]. In order to exchange energy and create the possibility of a Hopf bifurcation in the system, the minimal model should contain at least a flexural (heave) and a torsional (pitch) mode. The SMA nonlinear behaviour is described by an heuris-

\footnotetext{
a e-mail: arnaud.malher@ensta-paristech.fr
}

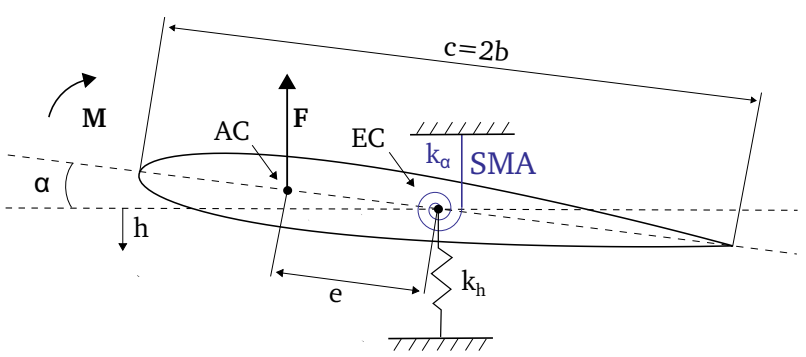

Fig. 1. 2DOFs Airfoil

tic model where the proeminent parameters are left free to vary. Numerical simulations are then conducted in order to investigate the effect of the SMA on the LCO. In particular, it is shown that for certain parameter range, the SMA spring can lead to a significant decrease of the amplitude of the LCO.

\section{2DOFs airfoil model}

\subsection{Dynamic model}

The model system under consideration is shown in Fig. 1. A Lagrangian formulation is used to express the evolution of the altitude $h$ (heave) and the angle of attack $\alpha$ (pitch) [8]. The kinetic energy reads $\mathcal{T}=\frac{1}{2} m \dot{h}^{2}+\frac{1}{2} I_{\alpha} \dot{\alpha}^{2}+$ $S_{\alpha} \dot{h} \dot{\alpha}$, and the potential energy reads $\mathcal{V}=\frac{1}{2} k_{\alpha} \alpha^{2}+\frac{1}{2} k_{h} h^{2}$. In these expressions, $I_{\alpha}$ is the inertial moment and $S_{\alpha}$ the static moment, the structural coupling between torsional and flexural motions being fully quantified by the term $S_{\alpha} \dot{h} \dot{\alpha}$. The source terms $F$ and $M$ (aerodynamic force and moment) are classically derived from the lift coefficient $C_{L}$ such as $F=\frac{1}{2} \rho U^{2} S C_{L}$ and $M=e F$, where $\rho$ is the fluid density, $U$ the upstream airspeed and $S$ the airfoil section. We assume that the angle of attack remains small, so that the lift coefficient depends linearly on $\alpha$, so that 


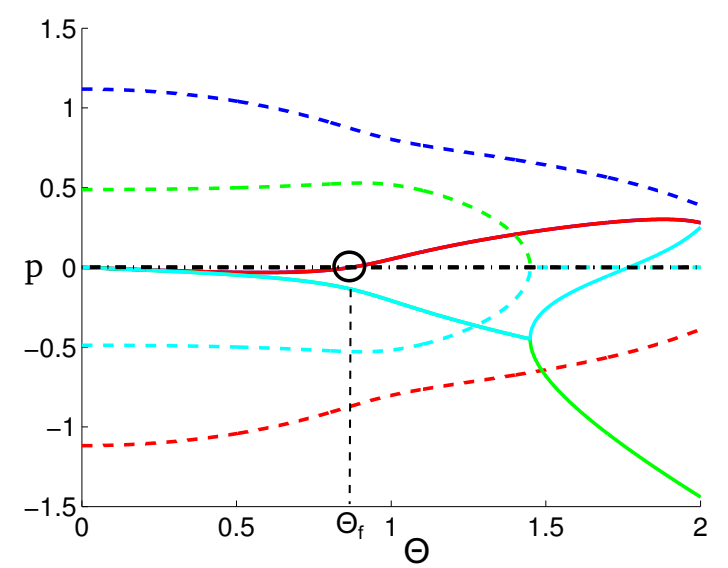

Fig. 2. Real (solid lines) and imaginary (dotted lines) parts of the solutions of the eigenvalue problem for Eq. (1) given as $\operatorname{det}(\mathbf{A})=$ 0 , with $\mathbf{A}$ defined in Eq. (2). Parameters of the problem listed in Table 3. $\Theta$ is a reduced velocity (see text).

$C_{L}=\left(\partial C_{L} / \partial \alpha\right) \alpha\left(\left.C_{L}\right|_{\alpha=0}=0\right.$ because the airfoil is symmetric). We consider a pseudo-static motion, i.e. the airfoil speed $\dot{h}$ is not neglected as compared to the upstream airspeed $U$. Lagrange equations lead to the following dynamical system :

$$
\begin{gathered}
{\left[\begin{array}{cc}
m & S_{\alpha} \\
S_{\alpha} & I_{\alpha}
\end{array}\right]\left[\begin{array}{c}
\ddot{h} \\
\ddot{\alpha}
\end{array}\right]+\left[\begin{array}{cc}
\frac{1}{2} \rho U S C_{L, \alpha} & 0 \\
-\frac{1}{2} e \rho U S C_{L, \alpha} & 0
\end{array}\right]\left[\begin{array}{c}
\dot{h} \\
\dot{\alpha}
\end{array}\right]+} \\
{\left[\begin{array}{cc}
K_{h} & \frac{1}{2} \rho U^{2} S C_{L, \alpha} \\
0 & K_{\alpha}-\frac{1}{2} e \rho U^{2} S C_{L, \alpha}
\end{array}\right]\left[\begin{array}{c}
h \\
\alpha
\end{array}\right]=0}
\end{gathered}
$$

The flutter velocity $U_{f}$, for which the flutter instability occurs, is determined from (1). Assuming that the airfoil motion is harmonic, $h=\tilde{h} \exp (p t)$ and $\alpha=\tilde{\alpha} \exp (p t)$ with $p \in \mathbb{C}$, replacing in Eq. (1) one finds

$$
\mathbf{A}\left[\begin{array}{c}
\tilde{h} \\
\tilde{\alpha}
\end{array}\right]=0, \text { with }
$$

$\mathbf{A}=\left[\begin{array}{cc}m p^{2}+\frac{1}{2} \rho U S C_{L, \alpha} p+K_{h} & S_{\alpha} p^{2}+\frac{1}{2} \rho U^{2} S C_{L, \alpha} \\ S_{\alpha} p^{2}-\frac{1}{2} e \rho U S C_{L, \alpha} p & I_{\alpha} p^{2}+K_{\alpha}-\frac{1}{2} e \rho U^{2} S C_{L, \alpha}\end{array}\right]$

Eq. (2) has non trivial solutions for $\operatorname{det}(\mathbf{A})=0$. This yields a polynomial of the fourth order in the variable $p$, the solutions of which are plotted in Fig. 2. $U_{f}$ is found as soon as the real part of a root crosses zero and becomes positive. An example is given in Fig. 2 which has been computed using the parameter values of Table 3 . The critical flutter velocity is found for that case as $\Theta_{f} \simeq 0.87$, where $\Theta$ is a reduced velocity such that $\Theta=\frac{U}{b} \sqrt{\frac{I_{\alpha}}{K_{\alpha}}}$ (see section 3 ).

\subsection{SMA model}

The SMA spring is assumed to behave in a pseudo-elastic manner, which is briefly recalled in Fig. 3. This nonlinear behaviour is characterized by a solid-solid phase change between two different states. The first one is called austenite, is stable at large temperatures and is the natural state of the spring at rest. The microstructure of the austenite phase

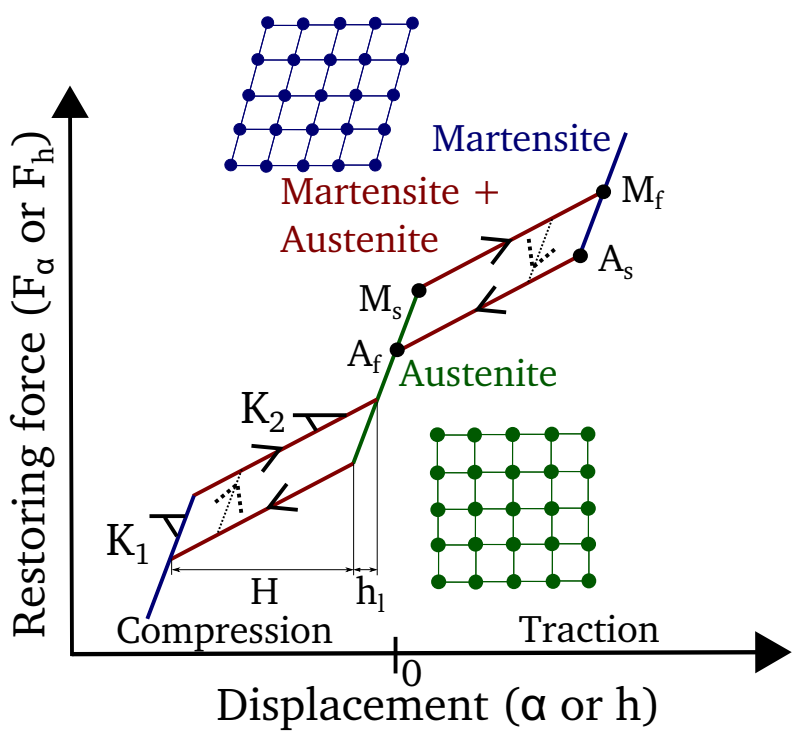

Fig. 3. Pseudo-elastic behaviour of SMA and its microstructure for each phase.

is also sketched in Fig. 3. When deformed, the microstructure of the SMA turns into a new phase, energetically stable at small temperatures, and for which the microstructure is now oriented, called the martensite phase. The points in the stress-strain space where the transformations start and finish are usually denoted with the subscripts $s$ (start) and $f$ (finish), so that for instance $M_{f}$ refers to the point where the martensitic transformation has been fully accomplished in the SMA structure. The path followed in the stress-strain relationship is not the same when the material is charged or decharged, thus an hysteresis loop appears as illustrated in Fig. 3. This hysteretic behaviour is the most salient feature of the pseudo-elastic behaviour of SMAs. The dissipated energy during a cycle is proportional to the area of the hysteresis loop so that the more the loop area is large, the more energy is prone to be dissipated in the device.

The nonlinear behaviour of single dof SMAs can be derived from a general, three-dimensional model infered from thermodynamical laws and then reduced by considering ad-hoc assumptions, see e.g. [6]. In this contribution, a simple heuristic model is used instead as it has the capacity to retrieve the main features of the dynamical behaviour within a light computational framework. It is built on the behaviour sketched in Fig. 3 by approximating each part of the diagram by a linear relationship. An internal auxiliary variable playing the role of the fraction of martensite is defined so as to keep the memory of the precedent state of the material in a dynamical simulation. For simplification, it is assumed that the slope of the purely austenitic and purely martensitic phases are the same, as well as the slopes during the reverse or transverse transformations, so that the main characteristics of the SMA are defined by $K_{1}$ and $K_{2}$ only as shown in Fig. 3. It is also assumed that the behaviour of the spring is symmetric in traction and compression. Internal loops are described following the sketch in Fig. 3. The SMA is then fully described with the following set of parameters illustrated in Fig. 3: $K_{1}, K_{2}, A_{f}$, $h_{l}$ and $H$. With these parameters, the loop area is equal to 


\begin{tabular}{cccccc}
\hline$r_{\alpha}$ & $\mu$ & $x_{\alpha}$ & $\Omega$ & $\gamma$ & $C_{L, \alpha}$ \\
\hline 0.5 & $1 / 10 \pi$ & 0.2 & 0.5 & 0.4 & $2 \pi$
\end{tabular}

Table 1. Non dimensional aeroelastic parameters.

$h_{l} H \sqrt{1+K_{1}^{2}} \sqrt{1+K_{2}^{2}} \cos \left(\arctan \left(1 / K_{1}\right)+\arctan \left(K_{2}\right)\right)$. Hence the control parameters of this loop are $h_{l}, H, K_{1}$ and $K_{2}$.

After the flutter instability, the wing experiences largeamplitude motions especially on the pitch mode, whereas the amplitude of the motion of the heave mode remains fairly small. In order to take advantage of the dissipative properties of the SMA, it appears logical to include a SMA spring on the torsional motion, whereas the flexural spring is left unchanged with a linear behaviour law.

\subsection{Final model}

The final model with a SMA torsional spring in order to control the pitch mode is derived by inserting the nonlinear behaviour of the SMA spring on the second equation of(1). It reads:

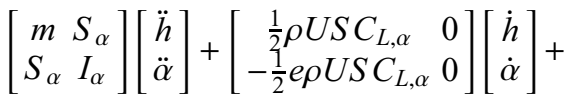

$$
\begin{aligned}
& {\left[\begin{array}{cc}
K_{h} & \frac{1}{2} \rho U^{2} S C_{L, \alpha} \\
0 & -\frac{1}{2} e \rho U^{2} S C_{L, \alpha}
\end{array}\right]\left[\begin{array}{l}
h \\
\alpha
\end{array}\right]=\left[\begin{array}{c}
0 \\
-F_{N L}^{S M A}(\alpha)
\end{array}\right],}
\end{aligned}
$$

where $F_{N L}^{S M A}$ is the nonlinear restoring force of the SMA depicted in Fig. 3.

The problem is then reduced to a nondimension system of equations

$$
\begin{aligned}
& {\left[\begin{array}{cc}
1 & x_{\alpha} \\
x_{\alpha} & r_{\alpha}^{2}
\end{array}\right]\left[\begin{array}{c}
y^{\prime \prime} \\
\alpha^{\prime \prime}
\end{array}\right]+\left[\begin{array}{cc}
\mu C_{L, \alpha} \Theta & 0 \\
-\mu \gamma C_{L, \alpha} \Theta & 0
\end{array}\right]\left[\begin{array}{c}
y^{\prime} \\
\alpha^{\prime}
\end{array}\right]+} \\
& {\left[\begin{array}{cc}
\Omega^{2} & \mu C_{L, \alpha} \Theta^{2} \\
0 & -\mu \gamma C_{L, \alpha} \Theta^{2}
\end{array}\right]\left[\begin{array}{c}
y \\
\alpha
\end{array}\right]=\left[\begin{array}{c}
0 \\
-f_{N L}^{S M A}(\alpha)
\end{array}\right],}
\end{aligned}
$$

$$
\begin{array}{lcc}
\text { with } \quad y=\frac{h}{b}, & \tau=\frac{K_{\alpha} t}{I_{\alpha}}, & ()^{\prime}=\frac{d}{d \tau}, \\
r_{\alpha}=\sqrt{\frac{I_{\alpha}}{m b^{2}}}, \mu=\frac{\rho b S}{2 m}, \quad x_{\alpha}=\frac{S_{\alpha}}{m b}, & \Theta=\frac{U}{b} \sqrt{\frac{I_{\alpha}}{K_{\alpha}}}, \\
\Omega=\sqrt{\frac{K_{h} I_{\alpha}}{m K_{\alpha}}} & \gamma=\frac{e}{b}, & \text { and } f_{N L}^{S M A}=\frac{F_{N L}^{S M A} I_{\alpha}}{m b^{2} K_{\alpha}}
\end{array}
$$

\section{Results and discussion}

The aeroelastic parameters for the two-dofs system have been selected according to [8], they are listed in Table 3. Eq. (4) is integrated in time with a fourth-order RungeKutta scheme. The initial condition is generally prescribed as a small perturbation on the heave mode. To gain insight on the critical parameter values, the flutter speed $\Theta_{f}$ can be derived analytically from the equations of motion. Referring to section 1 , one can use the fact that at the critical velocity $\Theta_{f}$, one of the real part of the roots vanishes. Assuming then $p$ purely imaginary, separating real and imaginary parts in the 4 th-order equation in $p$, and grouping the
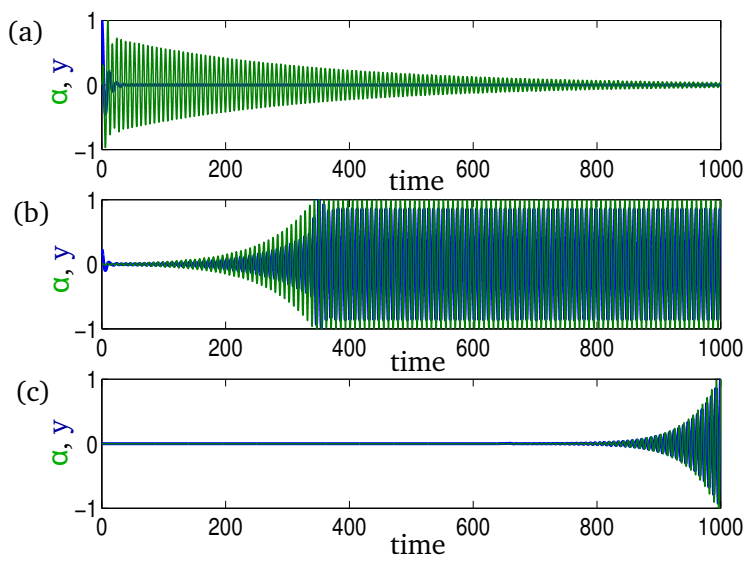

Fig. 4. Evolution of the heave $h$ (blue line) and pitch $\alpha$ (green line) as function of time, for three different reduced velocities: (a) $\Theta=0.86$ (b) $\Theta=0.91$ (c) $\Theta=0.93$. The curve are normalized to their maximal value.

terms to eliminate $p$, the following expression is found, for which $\Theta_{f}$ is solution

$$
\begin{aligned}
& A_{1} \Theta^{2}-A_{2}=0 \\
& \text { with }\left\{\begin{array}{l}
A_{1}=\mu C_{L, \alpha}\left(r_{\alpha}^{2}+\gamma x_{\alpha}\right) \\
A_{2}=r_{\alpha}^{2} x_{\alpha}
\end{array}\right. \\
& \text { thus } \Theta_{f}=\sqrt{\frac{A_{2}}{A_{1}}}=\sqrt{\frac{r_{\alpha}^{2} x_{\alpha}}{\mu C_{L, \alpha}\left(r_{\alpha}^{2}+\gamma x_{\alpha}\right)}}=0.87 \text {. }
\end{aligned}
$$

When $\Theta<\Theta_{f}$, the airfoil motion decreases and tends to zero, see Fig.4(a). When $\Theta \geq \Theta_{f}$, the flutter instability occurs and the position at rest is not stable anymore. However, the energy of the LCO can be dissipated by the SMA, so that for a certain range of reduced velocity, the amplitude of the motion saturates thanks to the nonlinear behaviour of the SMA, as illustrated in Fig.4(b). When the motion amplitudes of the LCO are beyond the end of the martensitic transformation, the potential of dissipation of the SMA is reached, so that divergent motions are retrieved. The critical speed above which the motion diverges again is denoted $\Theta_{c}$.

\subsection{Influence of SMA parameters}

The key parameter in order to optimize the passive control of the flutter instability is obviously $\Theta_{c}$, indeed the larger $\Theta_{c}$, the larger the range of flow velocities for which the LCO amplitude is controlled. However, due to intrinsic physical behaviour of the SMA, the loop area cannot be selected as large as theoretically desired. An important feature is given by $h_{l}$, indeed the smaller is $h_{l}$, the sooner the martensitic transformation will be activated so that the energy dissipation will occur rapidly. Let us investigate our last SMA parameter which is the ratio $K_{1} / K_{2}$, the LCO amplitude is plotted vs the airflow speed in Fig. 5 for different ratio $K_{1} / K_{2}$. These calculations are made by increasing and then decreasing the airflow speed step by step, a cubic stiffness is added to the model in order to saturate the system after the critical speed, the loop area is the same for all simulations. Some remarkable behaviour can be observed: 


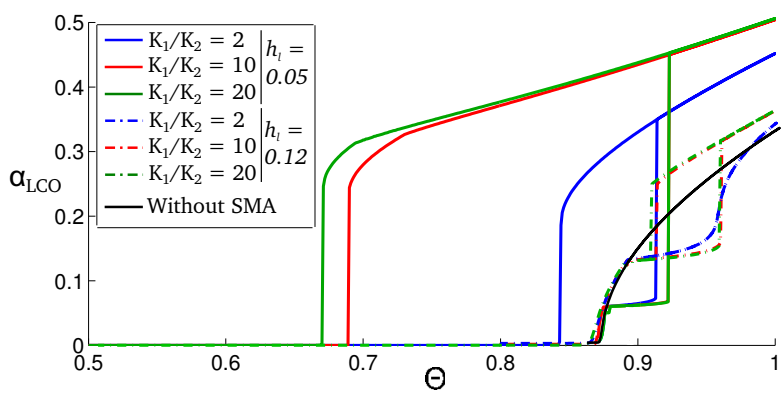

Fig. 5. Amplitude of the LCO for different SMA parameters

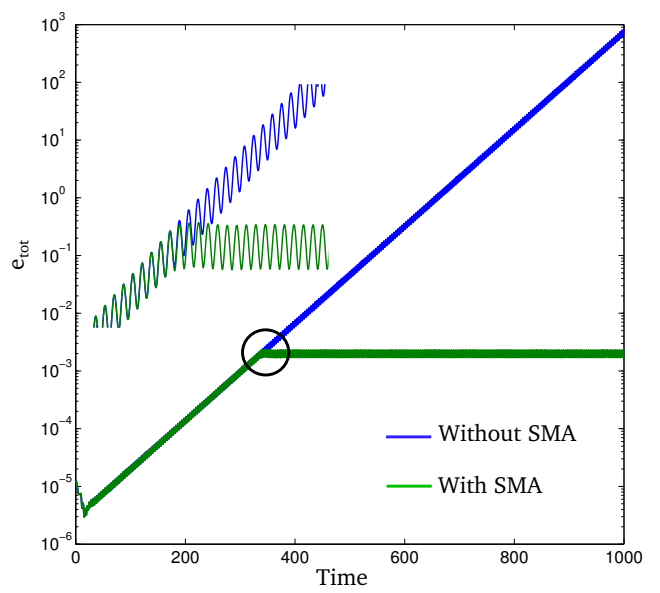

Fig. 6. Total energy $e_{\text {tot }}$ versus time for the aeroelastic system (parameters of Table 3), with and without SMA, and for $\Theta=0.9$.

- When $\Theta$ is decreasing, the LCO remains after $\Theta<\Theta_{f}$ for large ratio $K_{1} / K_{2}$ and small $h_{l}$.

- There is an important gap after $\Theta$ exceed $\Theta_{c}$ regardless of $h_{l}$ and $K_{1} / K_{2}$.

Thus one must pay attention to the critical velocity, because just as the upstream speed is equal to $\Theta_{c}$, the SMA will strongly increase the value of $\alpha_{L C O}$ and damage the structure.

\subsection{Energy exchange}

From section 2.1 the total energy reads

$$
E_{\text {tot }}=\frac{1}{2}\left(m \dot{h}^{2}+I_{\alpha} \dot{\alpha}^{2}+K_{\alpha} \alpha^{2}+K_{h} h^{2}\right)+S_{\alpha} \dot{h} \dot{\alpha} .
$$

Then the nondimensional energy is

$$
e_{t o t}=\frac{1}{2}\left(\dot{y}^{2}+r_{\alpha}^{2} \dot{\alpha}^{2}+k_{\alpha} \alpha^{2}+\Omega^{2} y^{2}\right)+x_{\alpha} \dot{y} \dot{\alpha} .
$$

Its evolution is plotted in Fig. 6 for system with and without SMA, for an airflow velocity $\Theta$ such that: $\Theta_{f}<\Theta<$ $\Theta_{c}$. For each cycle the energy increase of the system without SMA corresponds exactly to the energy dissipated by the SMA in its internal loop (for example right after the system with SMA enters in its first internal loop this loop dissipates $1.19810^{-4}$ and during this time the system without SMA grew $1.18910^{-4}$ ). Hence in this regime, the energy saturates to a finite value.

\section{Conclusion}

Whether one want to harvest energy or avoid devastating intabilities, the control of aeroelastic flutter is a critical issue. An option is discussed here by using SMA springs in pseudo-elastic behaviour, in order to use the potential of dissipation of such materials. A numerical study with a simple heuristic model for the behaviour of the SMA has shown that the amplitude of the LCO after the flutter velocity can be significantly reduced by adding a SMA spring on the pitch mode of the two-dofs aeroelastic system. However, once the damping capacity of the SMA is reached, the LCO amplitudes are larger than those obtained with a simple cubic spring. These findings have to be further investigated by considering different important effects of the SMA that have not been taken into account, in particular the dependence of the hysteresis loop on the frequency, as well as the asymmetry of the material behaviour. These preliminary findings will be confronted to experiments in order to confirm the potential of the SMA as a passive device for controlling the flutter instability. Preliminary results with aeroelastic parameters fitted to the experiments reported in [9] show an important increase of $\Theta_{c}$ so that the LCO amplitudes should be controlled on a larger range of flow velocities.

\section{References}

1. R. L. Bisplinghoff, H. Ashley, and R. L. Halfman. Aeroelasticity. Dover Publications, 1996.

2. S. Saadat, J. Salichs, M. Noori, Z. Hou, H. Davoodi, I. Bar-On, Y. Suzuki, and A. Masuda. An overview of vibration and seismic applications of NiTi shape memory alloys. Smart Materials and Structures, 11(2):218, 2002.

3. J.K. Strelec, D.C. Lagoudas, M.A. Kahn, and J. Yen. Design and implementation of a shape memory alloy actuated reconfigurable airfoil. Journal of Intelligent Material Systems and Structures, 14(4-5):257273, 2003.

4. W. Lacarbonara, D. Bernardini, and F. Vestroni. Nonlinear thermomechanical oscillations of shape-memory devices. International Journal of Solids and Structures, 41(5-6):1209 - 1234, 2004.

5. D. Bernardini and G. Rega. The influence of model parameters and of the thermomechanical coupling on the behavior of shape memory devices. International Journal of Non-Linear Mechanics, 45(10):933 - 946, 2010.

6. M. Ould Moussa, Z. Moumni, O. Doaré, C. Touzé, and W. Zaki. Non-linear dynamic thermomechanical behaviour of shape memory alloys. Journal of Intelligent Material Systems and Structures, 23(14):1593 - 1611, 2012.

7. O. Doaré, A. Sbarra, C. Touzé, M. Ould Moussa, and Z. Moumni. Experimental analysis of the quasistatic and dynamic torsional behaviour of shape memory alloys. International Journal of Solids and Structures, 49(1):32-42, 2011.

8. E. H. Dowell et al. A Modern Course in Aeroelasticity. Kluwer Academic, 1994.

9. X. Amandolese, S. Michelin, and M. Choquel. Low speed flutter and limit cycle oscillations of a twodegree-of-freedom flat plate in a wind tunnel. Journal of Fluids and Structures, 43:244-255, 2013. 